\title{
Procedimiento de Collis-Nissen laparoscópico para el tratamiento del esófago corto en niños
}

\section{Laparoscopic Collis-Nissen approach for shortened esophagus treatment in children}

\author{
Carlos García-Hernández ${ }^{1,2 *}$, Lourdes Carvajal-Figueroa ${ }^{1,2}$, Christian Archivaldo-García ${ }^{1,2}$ y \\ Sergio Landa-Juárez ${ }^{1,2}$

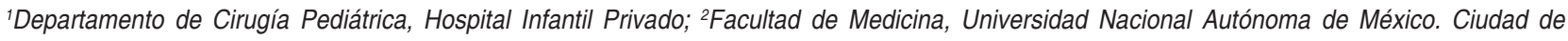 \\ México, México
}

\section{Resumen}

Antecedentes: El esófago corto es la imposibilidad de obtener una porción adecuada de esófago abdominal, por lo que se requiere alguna técnica de alargamiento. La mejor opción es el procedimiento de Collis. Objetivo: Demostrar la eficacia del procedimiento de Collis-Nissen por laparoscopía en niños. Método: Estudio retrospectivo, descriptivo, serie de casos, niños con reflujo y esófago corto, esofagoplastía de Collis con engrapadora y funduplicatura. Se analizaron edad, sintomatología, antecedentes quirúrgicos, tiempo quirúrgico, inicio de vía oral, tiempo de hospitalización, complicaciones y control del reflujo. Resultados: De 2005 a 2017 se trataron ocho niños de 4 a 15 años. De ellos, tres con antecedente de funduplicatura deslizada y dos con antecedente de atresia esofágica. El resto sin antecedentes, dos con estenosis. Síntomas; tos 8/8, dolor abdominal 5/8, disfagia 3/8. Sin complicaciones. Inició de vía oral al quinto día. Seguimiento de hasta 10 años, con remisión total de la sintomatología en seis casos. Discusión: Debido a que el diagnóstico de esófago corto verdadero depende de los hallazgos transoperatorios, los cirujanos pediatras deben reconocer esta condición al momento de practicar cualquier procedimiento antirreflujo. El procedimiento de Collis-Nissen laparoscópico es una opción segura y eficaz en estos pacientes.

Palabras Clave: Collis. Esofagoplastía. Laparoscopía.

\begin{abstract}
Background: Short esophagus is a disability to obtain a proper portion of abdominal esophagus, thus a lengthening technique is required. Collis approach is the best option. Objective: To demonstrate effectiveness of laparoscopic Collis-Nissen approach in children. Method: Retrospective and descriptive case series performed in children with reflux and short esophagus, Collis esophagoplasty was carried out with stapler, together with fundoplication. Age, symptomatology, surgical background, oral nutrition beginning, hospital stay, complications and reflux control were recorded. Results: Eight children, 4-15 years old were treated from 2005 to 2017. Three of them with slipped fundoplication background and two with esophageal atresia. The rest of the children had no background, two of them with stenosis. Symptoms; cough 8/8, abdominal pain 5/8, dysphagia 3/8. Without complications. Oral nutrition beginning at the $5^{\text {th }}$ day. Up to 10 years follow-up, with complete remission of the symptomatology in 6 years. Discussion: Since a true short esophagus diagnosis depends on transurgical findings, pediatric surgeons should notice this entity when practicing any antireflux procedure. Laparoscopic Collis-Nissen approach is safe and efficient in these patients.
\end{abstract}

Key Words: Collis. Esophagoplasty. Laparoscopy.

\section{Correspondencia:}

*Carlos García-Hernández

Circuito Valle Dorado, 12, Col. Loma de Valle Escondido

C.P. 52930, Atizapán de Zaragoza, Edo. de México, México

Fecha de recepción: 14-10-2018

Fecha de aceptación: 06-04-2019

E-mail: carloscirped@ hotmail.com

DOI: $10.24875 / C I R U .19000807$
Cir Cir. 2020;88(1):15-19

Contents available at PubMed www.cirugiaycirujanos.com 0009-7411/@ 2019 Academia Mexicana de Cirugía. Publicado por Permanyer. Éste es un artículo open access bajo la licencia CC BY-NC-ND (http://creativecommons.org/licenses/by-nc-nd/4.0/). 


\section{Introducción}

El esófago corto se define como la imposibilidad para obtener una porción de esófago abdominal de 2-3 cm, sin tensión, durante la disección para la construcción de algún procedimiento antirreflujo $0^{1,2}$.

Horvath, et al. ${ }^{3}$ definieron tres subtipos: esófago corto aparente, esófago corto verdadero reductible y esófago corto verdadero no reductible. En el primer tipo, el esófago tiene una longitud normal y después de una disección abdominal se obtienen $2-3 \mathrm{~cm}$ sin tensión. En el tipo 2 se requiere una disección mediastinal extensa $(5 \mathrm{~cm})$ para restaurar la unión esofagogástrica 2-3 cm por abajo del diafragma. En el tipo 3 , esto no es posible a pesar de una disección mediastinal extensa y se requiere algún procedimiento de alargamiento esofágico ${ }^{3}$.

La prevalencia en adultos varía del 8 al $10 \%$ en los pacientes a quienes se les practica una funduplicatura. En los niños se desconoce su incidencia, pues las publicaciones en pacientes pediátricos son escasas ${ }^{4,5}$.

La mayor parte de los esófagos cortos son adquiridos y están relacionados con la presencia de reflujo gastroesofágico, compuesto de ácido, bilis y jugos pancreáticos, que permiten que los iones de hidrógeno penetren en las capas profundas de la pared del esófago y produzcan edema, cicatrización, fibrosis y contracción cicatricial de la capa longitudinal muscular, con acortamiento intrínseco del esófago $0^{2,6,7}$.

El tratamiento del esófago corto está encaminado hacia el control del reflujo gastroesofágico; si se encuentra asociado con estenosis requiere que se practiquen, además, dilataciones esofágicas después de haber resuelto el reflujo.

Para poder construir una adecuada funduplicatura es indispensable:

- Determinar la altura de la unión esofagogástrica.

- Asegurarse de tener 2-3 cm de esófago intraabdominal sin tensión

- Si lo anterior no es posible a pesar de una amplia disección mediastinal, se deberá llevar a cabo algún procedimiento que permita mantener la funduplicatura por abajo del diafragma sin tensión.

Se ha propuesto subir el diafragma por arriba de la funduplicatura en un procedimiento llamado hiatoplastía extendida 4 .

Sin embargo, la mejor opción es la ideada por Collis en 1957, en la que se utiliza la curvatura menor del estómago para alargar el esófago ${ }^{8,9}$. La factibilidad y las ventajas de llevar a cabo dicho procedimiento

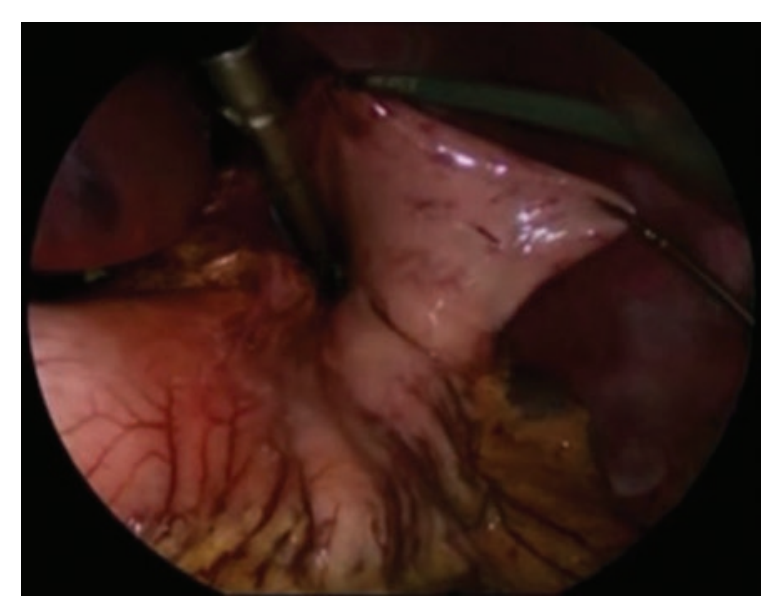

Figura 1. Colocación de engrapadora para el alargamiento esofágico.

mediante abordaje por cirugía de mínima invasión en adultos han sido demostradas ${ }^{2,7-9}$.

El objetivo del presente trabajo es demostrar la eficacia del procedimiento de Collis-Nissen por un abordaje de mínima invasión abdominal para el tratamiento del esófago corto asociado a reflujo gastroesofágico en niños.

\section{Método}

Estudio retrospectivo, descriptivo, serie de casos, en el que se incluyeron pacientes pediátricos con enfermedad por reflujo gastroesofágico y con un esófago corto verdadero que requirieron un procedimiento de alargamiento esofágico (tipo 3 de Horvath).

A todos los pacientes se les practicó disección esofágica por laparoscopía, mediante abordaje de cuatro puertos, con óptica de $5 \mathrm{~mm}$ y $30^{\circ}$, y presión de $10 \mathrm{mmHg}$, insuflando $1 \mathrm{l} / \mathrm{min}$, y endoscopía digestiva en ese momento para determinar el sitio de la unión esofagogástrica.

En los pacientes en los que no fue posible obtener $2 \mathrm{~cm}$ de esófago abdominal a pesar de una disección mediastinal extensa $(5 \mathrm{~cm})$ se practicó un procedimiento de Collis a través de una incisión abdominal en el epigastrio. Sin trocar se introdujo una engrapadora endoscópica lineal de $45 \mathrm{~mm}$, que se colocó en la unión esofagogástrica nativa, para alargar el esófago con la curvatura menor del estómago (Figs. 1 y 2). Se asoció con un procedimiento de Nissen al rodear este nuevo esófago con el nuevo fondo gástrico $360^{\circ}$, colocando tres suturas de poliéster 2-0 (Fig. 3). Previamente se practicó plastia de pilares con poliéster 2-0.

En los pacientes con estenosis se realizó además gastrostomía videoasistida. A todos se les practicó estudio contrastado al quinto día de la operación y al mes. 


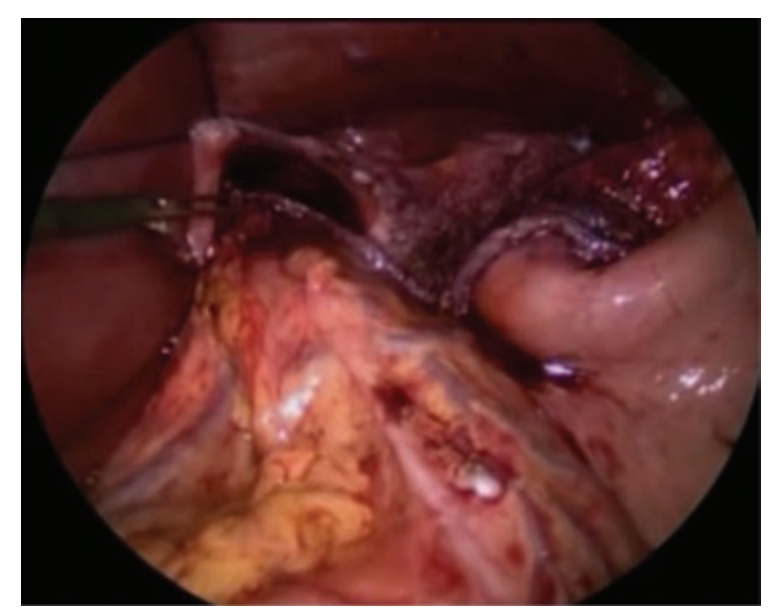

Figura 2. Apariencia del neoesófago de $4.5 \mathrm{~cm}$.

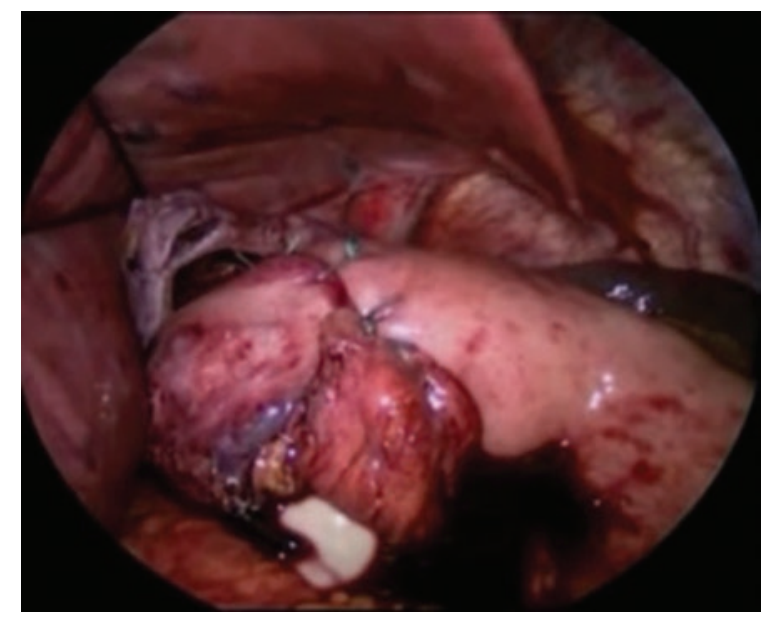

Figura 3. Creación de la nueva funduplicatura alrededor de la porción de esófago alargada.

Se realizó a los pacientes una endoscopía de control. A los que tenían también estenosis se les practicó dilatación esofágica con sonda tipo Savary en la segunda semana del posoperatorio.

Se analizaron la edad, el sexo, el peso, la sintomatología, los antecedentes quirúrgicos, la presencia de estenosis, el tiempo quirúrgico, el sangrado, las fistulas, el inicio de la vía oral, el tiempo de hospitalización y las complicaciones.

\section{Resultados}

De 2005 a 2017 se trataron ocho pacientes (cinco hombres y tres mujeres), cuyas edades variaron de 4 a 15 años,.

A tres niños se les había practicado una funduplicatura laparoscópica que presentó deslizamiento con la presencia de una hernia paraesofágica. Las edades de estos pacientes fueron 5, 7 y 9 años. La cirugía antirreflujo se había practicado 6, 8 y 14 meses antes de esta valoración. Estuvo indicada en los tres niños por falla del tratamiento médico en presencia de reflujo gastroesofágico, con manifestaciones respiratorias y neumonías de repetición. Los síntomas que volvieron a presentar los pacientes fueron tos, pirosis, dolor abdominal y vómito, con un tiempo de evolución de 2, 3 y 6 meses, respectivamente. La serie esofagogastroduodenal mostró migración de la funduplicatura, con la presencia de una hernia paraesofágica. La endoscopía preoperatoria mostró una imagen compatible con una hernia paraesofágica con desplazamiento de la funduplicatura en los tres niños.

Dos pacientes tenían antecedente de atresia esofágica tipo $\mathrm{C}$, sin malformaciones asociadas, tratados con anastomosis primaria sin complicaciones, realizadas en el periodo neonatal. Al momento del diagnóstico del esófago corto, los pacientes tenían 4 y 5 años. Ambos presentaron reflujo gastroesofágico, con presencia de regurgitaciones, pirosis, sialorrea, irritabilidad, dolor abdominal y tos, que habían sido tratados con procinéticos y antiácidos durante 14 y 18 meses. La serie esofagogastroduodenal mostró estenosis en el sitio de la anastomosis esofágica en un paciente y presencia de reflujo gastroesofágico en el estudio dinámico en ambos pacientes. La endoscopía preoperatoria mostró estenosis en el sitio de la anastomosis en un paciente que permitió el paso del endoscopio, así como esofagitis y línea $Z$ por arriba del pinzamiento diafragmático en ambos pacientes.

Dos pacientes aparentemente sanos, sin antecedentes quirúrgicos ni de ingestión de algún cáustico, cursaron con disfagia progresiva, con un tiempo de evolución de la sintomatología de entre 9 y 12 meses. El estudio contrastado evidenció estenosis del tercio inferior del esófago (Fig. 4). La endoscopía preoperatoria corroboró la estenosis del esófago con presencia de esofagitis (Fig. 5).

El otro paciente, sin antecedentes quirúrgicos, tenía sintomatología de reflujo gastroesofágico, de 2 años de evolución, caracterizada por tos, broncoespasmo, pirosis y sialorrea, que recibió diferentes tratamientos médicos sin respuesta. Se realizó una serie esofagogastroduodenal que mostró la presencia de una hernia hiatal por desplazamiento, que se corroboró por endoscopía.

A todos se les practicó pH-metría con impedanciometría, que reportó la presencia de reflujo ácido patológico en todos los pacientes. 
El diagnóstico de esófago corto verdadero se estableció mediante endoscopía transoperatoria en todos los pacientes.

Se obtuvieron en promedio $4.5 \mathrm{~cm}$ de neoesófago. El tiempo quirúrgico varió de 120 a 150 minutos. No hubo complicaciones transoperatorias. Sin dehiscencia ni fístulas, se inició la vía oral al quinto día con líquidos claros y dieta en papillas en todos los pacientes, con adecuada tolerancia. El tiempo de hospitalización varió de 6 a 8 días.

En los tres pacientes con estenosis, estas se resolvieron con dos sesiones de dilataciones en dos pacientes y con cuatro sesiones de dilataciones en otro paciente, iniciadas el día 15 del posoperatorio, llevando a cabo una sesión de dilatación por semana. En ninguno de estos niños se presentó recidiva de la estenosis.

El mayor tiempo de seguimiento fue de 10 años en un paciente y el menor tiempo fue de 8 meses, que es el tiempo transcurrido desde la operación hasta el momento de preparar esta publicación.

En seis pacientes con un seguimiento mínimo de 1 año desapareció la sintomatología de reflujo gastroesofágico, toleran adecuadamente la vía oral y no reciben ningún medicamento antiácido.

Dos pacientes, con antecedente de migración de la funduplicatura y que fueron operados de Collis-Nissen 12 y 20 meses antes de esta publicación, aún manifiestan dolor abdominal y pirosis ocasional que mejora con la administración de antiácidos.

A todos los pacientes se les practicó una endoscopía de control a los 6 meses y a siete niños a los 12 meses del posoperatorio, sin evidencia de estenosis. En los pacientes que la habían presentado, o inflamación por reflujo en todos, así mismo hubo competencia de la nueva unión esofagogástrica e integridad de la funduplicatura durante la maniobra endoscópica de versión (Fig. 6).

\section{Discusión}

La funduplicatura de Nissen controla la enfermedad por reflujo gastroesofágico en el 95\% de los pacientes. Para su correcta realización, el fondo gástrico se debe colocar 2-3 cm por arriba de la unión esofagogástrica ${ }^{10}$.

Cuando no es posible obtener $3 \mathrm{~cm}$ de esófago abdominal sin tensión a pesar de una disección mediastinal amplia, debe reconocerse que se trata de un esófago corto adquirido verdadero ${ }^{2,3}$ que requiere alguna forma de tratamiento diferente, que controle el reflujo

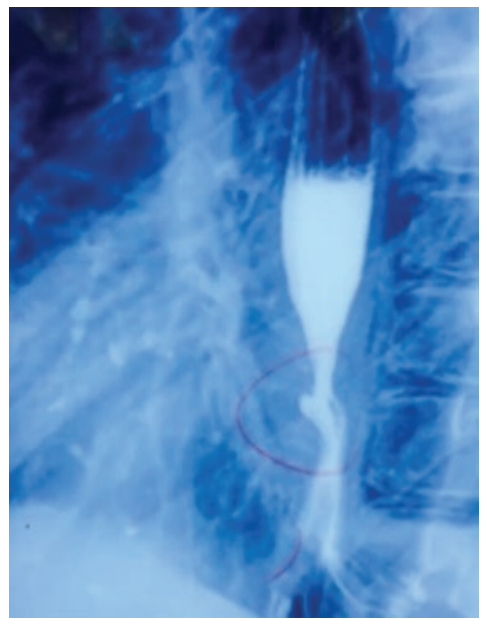

Figura 4. Estudio contrastado preoperatorio en el que se aprecia la estenosis del esófago.

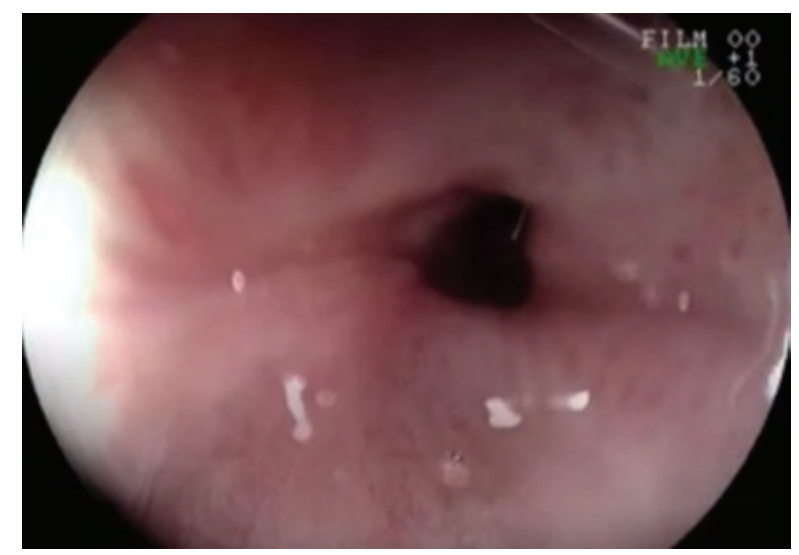

Figura 5. Imagen endoscópica de la estenosis preoperatoria.

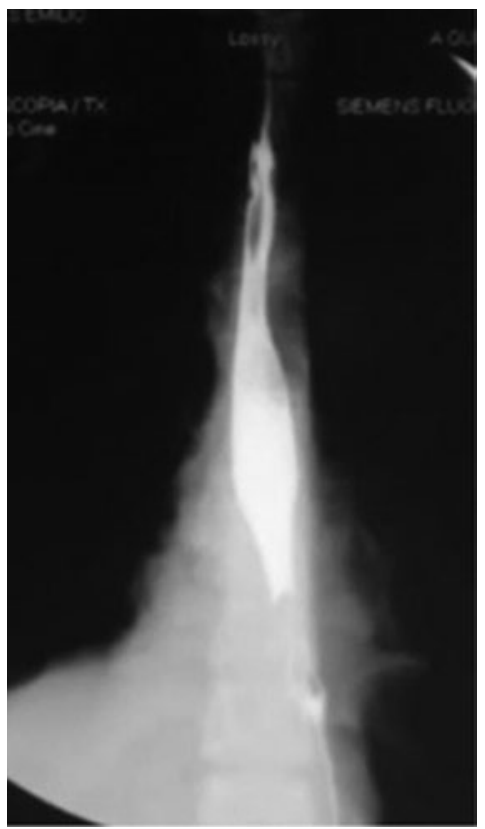

Figura 6. Estudio contrastado posoperatorio con resolución de la estenosis. 
gastroesofágico, tal como se estableció en los pacientes de este trabajo.

Existen numerosas publicaciones de tratamiento del esófago corto en adultos, pero en niños son escasas, por lo que la frecuencia de este padecimiento en pacientes pediátricos se desconoce ${ }^{4,5}$.

Collis ${ }^{8}$ publicó en 1957 una técnica en la que se alarga el esófago con la curvatura menor del estómago, que posteriormente se complementó con un procedimiento antirreflujo tipo Nissen.

Este procedimiento se ha realizado de manera segura mediante abordajes mínimamente invasivos en pacientes adultos ${ }^{9}$. Vargas, et al..$^{11}$ reportaron esta técnica para pacientes con atresia de esófago en cirugía abierta, en tanto que Rothenberg y $\mathrm{Chin}^{5}$ publicaron la aplicación de esta técnica por abordaje laparoscópico, con buenos resultados, en niños con reflujo gastroesofágico y esófago corto asociado a atresia de esófago.

Encontramos, en este trabajo, que esta técnica también es útil cuando el esófago corto se presenta en niños con funduplicaturas deslizadas y en pacientes con reflujo gastroesofágico grave asociado a estenosis.

En los adultos, esta operación requiere un abordaje combinado que comprende la introducción de la engrapadora por el tórax.

En este trabajo demostramos que es posible realizar este procedimiento de manera segura por un abordaje exclusivamente abdominal, lo que favorece una mejor recuperación.

El diagnóstico de esófago corto verdadero depende de los hallazgos transoperatorios. Los cirujanos pediatras debemos reconocer esta situación al momento de practicar cualquier procedimiento antirreflujo para poder ofrecer el tratamiento adecuado y prevenir el desplazamiento de la funduplicatura.

En este trabajo encontramos que el procedimiento de Collis-Nissen practicado mediante un abordaje de mínima invasión exclusivamente abdominal asociado al uso de suturas mecánicas no presentó complicaciones, como sangrado o dehiscencia de la línea de suturas. Además, también hallamos que este procedimiento controló la sintomatología producida por el reflujo.
El control de la enfermedad sin presencia de complicaciones demuestra que el procedimiento de CoIlis-Nissen es un procedimiento seguro y eficaz en el tratamiento del reflujo en los niños con esófago corto.

\section{Conflicto de intereses}

Los autores declaran que no existe conflicto de intereses.

\section{Responsabilidades éticas}

Protección de personas y animales. Los autores declaran que para esta investigación no se han realizado experimentos en seres humanos ni en animales.

Confidencialidad de los datos. Los autores declaran que han seguido los protocolos de su centro de trabajo sobre la publicación de datos de pacientes.

Derecho a la privacidad y consentimiento informado. Los autores han obtenido el consentimiento informado de los pacientes y/o sujetos referidos en el artículo. Este documento obra en poder del autor de correspondencia.

\section{Bibliografía}

1. Spechler SJ, Goyal RK. The columnar-lined esophagus, intestinal metaplasia, and Norman Barrett. Gastroenterology. 1996;110:614-21.

2. Hoang CD, Koh PS, Maddaus MD. Short esophagus and esophageal stricture. Surg Clin N Am. 2005;85:433-51.

3. Horvath KD, Swanstrom LL, Jobe BA. The short esophagus: pathophysiology, incidence, presentation, and treatment in the era of laparoscopic antireflux surgery. Ann Surg. 2000;232:630-40.

4. Bustorff-Silva J, Moreira APP, Cavalaro MA, Melo Filho AA. Extended hiatoplasty: early experience with a simple technique to increase the intra-abdominal esophageal length in complicated gastroesophageal reflux. J Pediatr Surg. 2001;36:555-8.

5. Rothenberg SS, Chin A. Laparoscopic Collis-Nissen for recurrent severe reflux in pediatric patients with esophageal atresia and recurrent hiatal hernia. J Lap Adv Surg Tech. 2010;20:787-90.

6. Gozzeti G, Pilotti V, Spangaro M, Bassi F, Grigioni W, Carulli N, et al. Pathophisiology and natural history of acquired short esophagus. Surgery. 1987;102:507-14.

7. Yau P, Watson DI, Jamieson GG, Myers J, Ascott N. The influence of esophageal length on outcomes after laparoscopic fundoplication. J Am Coll Surg. 2000;191:360-5.

8. Collis JL. An operation for hiatus hernia with short oesophagus. Thorax. 1957;12:181-8.

9. Richardson JD, Richardon RL. Collis-Nissen gastroplasty for shortened esophagus: long-term evaluation. Ann Surg. 1998;227:735-42.

10. Lobe TE, Schropp KP, Lunsford K. Laparoscopic Nissen fundoplication in childhood. J Pediatr Surg. 1993;28:358-61.

11. Vargas-Gómez MA, Méndez-Martínez D, Andrade-Sepúlveda V. Sustitución de esófago con técnica de Scharli y Collis-Nissen. Rev Mex Cir Pediatr. 2008;15:130-5. 